\title{
The emerging dynamic view of proteins: Protein plasticity in allostery, evolution and self-assembly
}

The dynamics of proteins has always been at the center of the interest of protein researchers, since it is intimately related with their function. Nevertheless, the emphasis on the uniqueness of the native structure, which is to a large extent encoded in the amino acid sequence of the protein, as beautifully demonstrated by Anfinsen experiments [1], contributed to the development of a "static" view of proteins. This can be exemplified through three widely used simplifications: (1) The one-sequence-one-structure-one-function paradigm. The uniqueness of protein structure is a premise of protein structure prediction methods, critically assessed in the CASP experiment [2]. (2) The two-states model of protein folding in which only the native and the denatured states are thermodynamically relevant. An inspection of the ProTherm database [3] suggests that a large number of folding experiments have been fitted using the two-states model despite the fact that data favor more complex models. (3) The assumption that the fold is almost always conserved during evolution, on which databases of protein structure classification $[4,5]$ are based.

Recently, a more dynamic view of proteins is gaining the attention of researchers again. This change of point of view has been, in our opinion, mainly influenced by the following developments: (i) the appraisal that a large fraction of the proteins of complex organisms are unstructured in their native state, and that this structural disorder, far from being a bizarre property, is crucial for their regulatory function [6]; (ii) the reappraisal of allostery, i.e. the ability of proteins to propagate conformational changes from a control site to a distant active site, as an intrinsic property of protein dynamics that is deeply related with enzymatic catalysis [7,8]; (iii) the increasing number of experimental and theoretical studies on phosphorylation and other post-translational modifications, which are used by the cell to achieve allosteric control of protein structure and function [9]; (iv) the discovery that changes of protein structure during evolution are more common than previously thought [10-13] and that the interplay between intrinsic protein dynamics and structure change can favor protein evolvability [14,15]; (v) the clinical and possibly biological importance of proteins that have radically different alternative folded states, such as the prions $[16,17]$.

From the theoretical point of view, the energy landscape theory of protein folding $[18,19]$ has provided since the 1980s a framework for describing proteins as a statistical mechanical ensemble of different conformations. Nevertheless, the difficulty to determine "realistic" energy functions that reliably describe the energy landscape of proteins has shifted the focus to native-state based models, called Go models in the jargon of the field [20]. These Go models have often been studied in the two-states approximation of protein folding. The discovery that they can provide a wealth of information on the intrinsic dynamics of the native state through the harmonic approximation [21] (the so-called elastic network model), and that the predictions of these simplified models are in quantitative agreement with experimentally observed functional motions, has provided researchers with a powerful analytical tool to investigate the functional dynamics of proteins [22] that complements classical molecular dynamics [23].

The interplay between all these aspects has led to considerable progress in the understanding of how proteins are built and function. In our opinion, this progress is shaping a new view of proteins. The workshop "The emerging dynamic view of proteins: Protein plasticity in allostery, evolution and self-assembly", funded and hosted by the MPI for the Physics of Complex Systems in Dresden, Germany, was inspired by this idea, and it was aimed at favoring the dialogue between different disciplines focused around protein plasticity. The workshop was organized into six topical sections: (1) Allostery, with talks by Dorothee Kern, on experiments and computations that describe protein dynamics during catalysis; Devarajan Thirumalai, on the modeling of allosteric transitions in molecular machines; Igor Berezovsky, on a coarse-grained model of allosteric regulation and communication; Ammon Horovitz, on theory and experiments that describe the allosteric motion of the chaperonin GroEL; Thomas Weikl, on the role of conformational selection and induced fit in protein function; Vincent Hilser, illustrating that biological ensembles are poised to respond; and Ruth Nussinov, describing structural networks of signaling pathways on the proteome scale. (2) Self-Assembly and aggregation, with talks by Simon Alberti, illustrating protein self-assembly and the formation of cellular structures and memories; Petra Schwille, describing spatial self-organization of the form of the Turing instability that drives morphogenesis, realized through a simple experiment of the reaction and diffusion of just two proteins; Joseph Marsh, illustrating how subunit flexibility facilitates protein complex assembly and evolution; and Fabrizio Chiti, reporting on the structural determinants of protein oligomer toxicity. (3) Structurally disordered proteins, with talks by Monica Fuxreiter, on ambiguity in protein interactions (fuzziness) and Vladimir Uversky, on intrinsic disorder-based interactions and their modulators. (4) Computational methods, with talks by Modesto Orozco, on protein dynamics at the genomic scale; Martin Zacharias, describing protein-protein docking methods that account for conformational changes; and Gunnar Schroeder, on protein structure and dynamics from low-resolution data. (5) Evolution of protein structure, function and dynamics, with talks by Erich Bornberg-Bauer, on the escape from adaptive conflict in the evolution of protein function through weak functional trade-offs and mutational robustness; Michael Lässig, on the mutation and selection balance in the evolution of viral proteins; Christine Orengo, on the plasticity of functional sites in evolving superfamiles; Nick Grishin, on the evolutionary changes of protein spatial structures; Rachel 
Kolodny, giving a bird's eye view of protein structure space; Arne Elofsson, reporting that disorder regions are indel hotspots; Jessica Stiltberg-Liberles, on the evolutionary dynamics of conformational flexibility in the prion protein and its remote homologs. (6) Folding, with talks by Oxana Galzitskaya, on the influence of organization of native structure on its folding, and Anna Tramontano, addressing the structural variability of antibodies with structure prediction techniques.

Furthermore, besides interesting contributed talks and poster sessions, the workshop had four discussion sections in which some of the invited speakers introduced the section's topic and a large number of participants expressed their views on the subject.

The first of these sections discussed the shift from single sequence-structure-function relationship to dynamic ensembles. There was consensus on the fact that the idea that the protein sequence determines the structure simplifies our view of proteins too much, and that dynamic ensembles constitute a description of proteins more suitable than PDB coordinates, however there was no agreement on which experimental techniques and mathematical descriptions are needed to proceed in this direction.

The second discussion addressed the relationship between structural disorder and protein complexity. In this case, there was no agreement on how we can quantify the structural complexity of a protein, and whether disordered proteins are more or less complex than enzymes that possess a unique structure and function. Proposers of the idea that disordered proteins are more complex argued that their sequences are characterized by a large number of splicing isoforms, their structures possess a larger number of different alternative conformations, and the large number of potential interaction partners that they have provides them with a multiplicity of functions. Moreover they often self-assemble to form complex molecular machines such as the centrosome and greatly contribute to the complexity of the organism. However, other participants argued that the sequences of disordered proteins are usually simpler than those of ordered proteins, in the sense that they tend to have high entropy, and they can be characterized as high entropy ensembles of structures.

In the third discussion section, on the evolution of protein structures, the question was whether we need a new view of protein structure space that explicitly recognizes that protein structures change in evolution. It was agreed that protein fold changes in evolution, as demonstrated through the work of Grishin [10] and others, and that these new ideas on protein structure and function evolution need to be and are being incorporated into structural classifications of proteins.

The fourth discussion addressed the question whether structural disorder is just an extreme version of the flexibility of folded proteins, which perform large conformational changes in their equilibrium dynamics, connecting folded sub-states that are relevant for their biological function. Elastic network models show that there is a deep relationship between the allosteric functional dynamics of proteins and their equilibrium dynamics in the native state. On the other hand, intrinsically disordered proteins often lack stable folded structures and they can be described only in terms of dynamical ensembles, therefore it is not clear whether it is possible and convenient to describe them with the same mathematical models used to describe the equilibrium dynamics of folded proteins.

A significant representation of the contributions and discussions presented at the workshop are reported in this special issue, which is organized in three main sections.

The first section is dedicated to "Allostery and collective functional movements". In this section, the group of Nussinov proposes that scaffolding proteins can actively control regulation and signaling of multienzyme complexes through allostery; Berezovsky reviews a method to predict functional sites through a coarse-grained model of allostery, and its possible application to the development of allosteric drugs; dos Santos et al. present a null model of protein conformational changes based on the elastic response in torsion angle space, proposing that conformational changes that significantly deviate from this null model have been optimized by natural selection; Ostermeir et al. present the application of advanced replica-exchange sampling to study the flexibility and plasticity of peptides and proteins; Valerianovna et al. propose that stability and rigidity/flexibility in protein folding are two sides of the same coin; and Weikl describes how conformational changes can affect catalysis, inhibition and drug resistance of enzymes with induced-fit binding mechanism such as the HIV-1 protease.

The second section, "structural flexibility in protein evolution", contains the contribution by Dessailly et al., who demonstrate that functional sites are extremely variable within a superfamily of evolutionarily related proteins; Light et al., who show that long indels in homologous eukaryotic proteins have a strong tendency to be disordered; and Moore et al., who quantify the evolution of modular proteins in a dense phylogenetic tree.

In the third section, "Disordered proteins and large macromolecular complexes", Labas et al. show that the optimization of the reorganization energy drives the evolution of the designed Kemp eliminase KE07; Malinovska et al. review the relationships between protein disorder, prion propensities, and self-organizing macromolecular collectives, suggesting that the extreme structural flexibility of disordered proteins is a key property for the self-organization of large macromolecular complexes; finally, the review by Uversky describes the biophysical properties that characterize intrinsically disordered proteins with respect to more familiar folded proteins.

\section{Acknowledgements}

We are grateful to the Max Planck Institute for the Physics of Complex Systems (MPIPKS) in Dresden, Germany, for generously hosting and financing the workshop "The emerging dynamic view of proteins", and to its staff, in particular Sabine Strecker, for taking care of all logistics, we thank all participants for the enthusiasm with which they took part in the workshop, and the editors of BBA for hosting this special issue.

\section{References}

[1] C.B. Anfinsen, Principles that govern the folding of protein chains, Science 181 (1973) 223-230.

[2] J. Moult, A decade of CASP: progress, bottlenecks and prognosis in protein structure prediction, Curr. Opin. Struct. Biol. 15 (2005) 285-289.

[3] M.M. Gromiha, H. Uedaira, J. An, S. Selvaraj, P. Prabakaran, A. Sarai, ProTherm, thermodynamic database for proteins and mutants: developments in version 3.0, Nucl. Acids Res. 30 (2002) 301-302.

[4] T.J.P. Hubbard, A.G. Murzin, S.E. Brenner, C. Chothia, SCOP: a Structural Classification of Proteins database, Nucleic Acids Res. 25 (1997) 236-239.

[5] C.A. Orengo, F.M.G. Pearl, G.E. Bray, A.E. Todd, A.C. Martin, L. Lo Conte, J.M. Thornton, The CATH Database provides insights into protein structure/function relationships, Nucleic Acids Res. 27 (1999) 275-279.

[6] V.N. Uversky, A.K. Dunker, Understanding protein non-folding, Biochim. Biophys Acta 1804 (2010) 1231-1264.

[7] A. del Sol, C.J. Tsai, B. Ma, R. Nussinov, The origin of allosteric functional modulation: multiple pre-existing pathways, Structure 17 (2009) 1042-1050.

[8] N.M. Goodey, S.J. Benkovic, Allosteric regulation and catalysis emerge via a common route, Nat. Chem. Biol. 4 (2008) 474-482.

[9] O.N. Jensen, Modification-specific proteomics: characterization of post-translational modifications by mass spectrometry, Curr. Opin. Chem. Biol. 8 (2004) 33-41.

[10] L.N. Kinch, N.V. Grishin, Evolution of protein structures and functions, Curr. Opin. Struct. Biol. 12 (2002) 400-408.

[11] W.R. Taylor, Evolutionary transitions in protein fold space, Curr. Opin. Struct. Biol. 17 (2007) 354-361.

[12] P.A. Alexander, Y. He, Y. Chen, J. Orban, P.N. Bryan, A minimal sequence code for switching protein structure and function, Proc. Natl. Acad. Sci. U. S. A. 106 (2009) 21149-21154.

[13] A. Pascual-Garcia, D. Abia, A.R. Ortiz, U. Bastolla, Cross-over between discrete and continuous protein structure space: insights into automatic classification and networks of protein structures, PLoS Comput. Biol. 5 (2009) e1000331.

[14] N. Tokuriki, D.S. Tawfik, Protein dynamism and evolvability, Science 324 (2009) 203-207. 
[15] J. Shorter, S. Lindquist, Prions as adaptive conduits of memory and inheritance, Nat. Rev. Genet. 6 (2005) 435-450.

[16] R.A. Moore, L.M. Taubner, S.A. Priola, Prion protein misfolding and disease, Curr. Opin. Struct. Biol. 19 (2009) 14-22.

[17] F. Chiti, C.M. Dobson, Protein misfolding, functional amyloid, and human disease, Annu. Rev. Biochem. 75 (2006) 333-366.

[18] J.D. Bryngelson, P.G. Wolynes, Spin glasses and the statistical mechanics of protein folding, Proc. Natl. Acad. Sci. U. S. A. 84 (1987) 7524-7528.

[19] J.N. Onuchic, Z. Luthey-Schulten, P.G. Wolynes, Theory of protein folding: the energy landscape perspective, Annu. Rev. Phys. Chem. 48 (1997) 545-600.

[20] C. Clementi, Coarse-grained models of protein folding: toy models or predictive tools? Curr. Opin. Struct. Biol. 18 (2008) 10-15.

[21] M.M. Tirion, Large amplitude elastic motions in proteins from a single-parameter, atomic analysis, Phys. Rev. Lett. 77 (1996) 1905-1908.

[22] I. Bahar, T.R. Lezon, L.W. Yang, E. Eyal, Global dynamics of proteins: bridging between structure and function, Annu. Rev. Biophys. 39 (2010) 23-42.

[23] J.L. Klepeis, K. Lindorff-Larsen, R.O. Dror, D.E. Shaw, Long-timescale molecular dynamics simulations of protein structure and function, Curr. Opin. Struct. Biol. 19 (2009) 120-127.

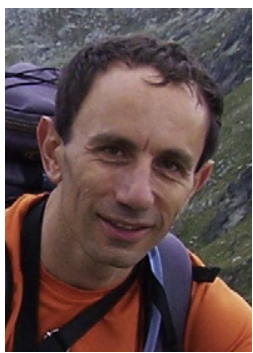

Ugo Bastolla studied physics in Rome with Luca Peliti and with Giorgio Parisi, turning since then his interest towards computational biology and evolution. During his post-doctoral stays in Germany with Peter Grassberger and with Ernst-Walter Knapp he studied simple models of protein folding, with the aim of predicting the stability of the folded state and model protein evolution with structural constraints. This line was subsequently expanded towards the influence of mutation bias, mutation rate and population size on the evolution of protein stability, the characterization of selective pressures in protein evolution, the evolution of protein structures and disordered proteins, and the intrinsic dynamics of proteins and its relationship with functional motions. His other main research interest, which he started while at the MPI of Golm (Germany), concerns the determinants of biodiversity in model ecosystems. He is currently applying these models to mutualistic networks and bacterial ecology. After some years in the center of astrobiology of Madrid, he is currently a researcher of the Spanish research council (CSIC) and directs the bioinformatics unit of the center of molecular biology Severo Ochoa in Madrid, Spain.

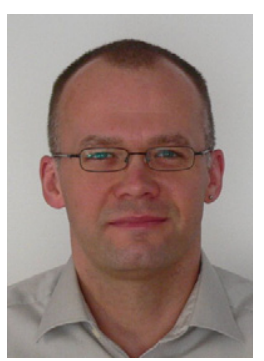

Markus Porto is a Heisenberg Fellow of the Deutsche Forschungsgemeinschaft at the Universität zu Köln. He has been at the Tel Aviv University, the Max Planck Institute for the Physics of Complex System (Dresden), and the Technische Universität Darmstadt. His research interest covers nonequilibrium systems, in particular as occurring in biology.

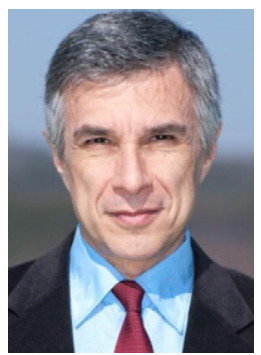

H. Eduardo Roman is currently a Visiting Professor at POSTECH working at the Division of IT Convergence Engineering. He has been at the Max Planck Institute for the Physics of Complex Systems (Dresden) and at the Department of Physics of the University of Milan-Bicocca. His interests cover different areas of Complex Systems such Bio-molecules, Nano-Materials, Fractals and Finance. $\mathrm{He}$ is a Fedeor Lynen Fellow of the Alexander von Humboldt Foundation.

Ugo Bastolla

Centro de Biología Molecular Severo Ochoa (CSIC-UAM), 28029 Madrid, Spain

Corresponding author. Tel.: + 34911964633. E-mail address: ubastolla@cbm.uam.es.

Markus Porto Institut für Theoretische Physik, Universität zu Köln, Zülpicher Str.77, 50937 Köln, Germany

H. Eduardo Roman Division of IT Convergence Engineering, POSTECH, San 31, Hyoja-Dong, Nam-Gu 790-784 Pohang, Gyungbuk, Republic of Korea 